\title{
Vitamin D Level, Respiratory Tract infections, and Controlled Trials : [Letter]
}

\section{Hemilä, Harri}

2009

Hemilä , H 2009 , ' Vitamin D Level, Respiratory Tract infections, and Controlled Trials :

[Letter] ' , Archives of Internal Medicine , vol. 169 , no. 15 , pp. 1443-1443 . https://doi.org/10.1001/archinternmed.20

http://hdl.handle.net/10138/228311

https://doi.org/10.1001/archinternmed.2009.246

publishedVersion

Downloaded from Helda, University of Helsinki institutional repository.

This is an electronic reprint of the original article.

This reprint may differ from the original in pagination and typographic detail.

Please cite the original version. 
Correspondence: Dr A. T. Yan, Division of Cardiology, St Michael's Hospital, Queen 6-030, 30 Bond St, Toronto, ON M5B 1W8, Canada (yana@smh.toronto.on.ca).

1. Yan AT, Yan RT, Huynh T, et al; Canadian Acute Coronary Syndrome Registry 2 Investigators. Understanding physicians' risk stratification of acute coronary syndromes: insights from the Canadian ACS 2 Registry. Arch Intern Med. 2009;169(4):372-378.

2. Yan AT, Yan RT, Tan M, et al. Risk scores for risk stratification in acute coronary syndromes: useful but simpler is not necessarily better. Eur Heart J. 2007; 28(9):1072-1078.

3. Yan AT, Yan RT, Tan M, et al; Canadian Acute Coronary Syndromes 1 and 2 Registry Investigators. Management patterns in relation to risk stratification among patients with non-ST elevation acute coronary syndromes. Arch Intern Med. 2007;167(10):1009-1016.

4. Lee CH, Tan M, Yan AT, et al; Canadian Acute Coronary Syndromes (ACS) Registry II Investigators. Use of cardiac catheterization for non-ST-segment elevation acute coronary syndromes according to initial risk: reasons why physicians choose not to refer their patients. Arch Intern Med. 2008;168(3):291296

5. Manfrini O, Bugiardini R. Barriers to clinical risk scores adoption. Eur Heart J. 2007:28(9):1045-1046.

\section{Vitamin D Level, Respiratory Tract Infections, and Controlled Trials}

$\mathbf{P}$

roponents of evidence-based medicine argue that conclusions of treatment effects should be based on controlled trials and not on observational studies. Nevertheless, based on the correlation between serum vitamin D level and infections, Ginde et al ${ }^{1}$ proposed that vitamin $\mathrm{D}$ supplementation may reduce the incidence of upper respiratory tract infections.

Nutrition is associated with a wide range of lifestyle variables, and there is a great risk of residual confounding caused by inadequate adjustments for complex social and environmental exposures acting over a person's life. ${ }^{2}$ Residual confounding may explain, for example, the divergent findings in observational studies and controlled trials on vitamin E. ${ }^{2}$ Therefore, great caution should be exercised when interpreting correlations between serum vitamin D levels and health outcomes.

Furthermore, certain randomized controlled trials on multivitamin supplements are relevant when considering the effect of vitamin D supplementation on respiratory tract infections. If a multivitamin supplement has no effect on infections, it seems justified to conclude that there is a lack of effect by each constituent of the supplement. In contrast, if a multivitamin supplement does have an effect, we cannot draw any specific conclusions because the effect can be caused by any single substance or the combination of several of them together. In this respect, the implications are quite different when the result of a multivitamin trial is positive or negative. Two multivitamin trials with 748 and 652 elderly participants included 160 and $200 \mathrm{IU} / \mathrm{d}$ of vitamin D in their supplements. ${ }^{3,4}$ The supplements had no effect on the incidence of upper or lower respiratory tract infections. ${ }^{3,4}$ Thus, following this logic, these trials indicate that increasing vitamin D intake has no effect on the incidence of respiratory tract infections in the elderly people studied.

Finally, the comment by Ginde et $\mathrm{al}^{1}$ on vitamin $\mathrm{C}$ is somewhat inaccurate. Although regular vitamin C (ascorbic acid) supplementation has not reduced the incidence of the common cold in ordinary people, it has con- sistently reduced the duration of colds that occurred during the supplementation period. In adults, the reduction in common cold duration was $8 \%$ and in children it was $13 \%{ }^{5}$ Therefore, we concluded in our Cochrane review that the therapeutic effects of vitamin $C$ should be further studied, in particular in children because no therapeutic trials with children have been carried out, although the effect of regular vitamin $C$ has been greater in them. ${ }^{5}$

Harri Hemilä, PhD, MD

Correspondence: Dr Hemilä, Department of Public Health, University of Helsinki, Mannerheimintie 172, PO Box 41, Helsinki FIN-00014, Finland (harri.hemila @helsinki.fi).

1. Ginde AA, Mansbach JM, Camargo CA Jr. Association between serum 25hydroxyvitamin D level and upper respiratory tract infection in the Third National Health and Nutrition Examination Survey. Arch Intern Med. 2009;169 (4):384-390.

2. Lawlor DA, Davey Smith G, Bruckdorfer KR, Kundu D, Ebrahim S. Those confounded vitamins: what can we learn from the differences between observational versus randomised trial evidence? Lancet. 2004;363(9422):1724-1727.

3. Liu BA, McGeer A, McArthur MA, et al. Effect of multivitamin and mineral supplementation on episodes of infection in nursing home residents: a randomized, placebo-controlled study [correction published in J Am Geriatr Soc 2007;55(3):478]. J Am Geriatr Soc. 2007;55(1):35-42.

4. Graat JM, Schouten EG, Kok FJ. Effects of daily vitamin E and multivitaminmineral supplementation on acute respiratory tract infections in elderly persons: a randomized controlled trial. JAMA. 2002;288(6):715-721.

5. Hemilä H, Chalker EB, Treacy B, Douglas RM. Vitamin C for preventing and treating the common cold. Cochrane Database Syst Rev. 2007;(3):CD000980.

\section{In reply}

We thank Dr Hemilä for this correspondence. The Cochrane review by Douglas et al ${ }^{1}$ supported our statement that vitamin $C$ has a limited role in prevention and treatment of colds and concluded that the prophylactic or therapeutic use of vitamin $C$ does not reduce the incidence or severity of colds. There was a modest reduction (approximately 12 hours) in duration of colds with prophylactic but not therapeutic use of vitamin C, but as those authors conclude, "there seems no justification for routine mega-dose vitamin $C$ supplementation in the normal population" and "therapeutic supplementation has not been shown to be beneficial."

We agree that observational data have limitations, particularly in nutrition research. As demonstrated by the vitamin E experience, ${ }^{2}$ randomized controlled trials may provide different conclusions than suggested by preceding observational studies, often because of incompletely controlled confounding. In addition, as we discussed, ${ }^{3}$ vitamin $D$ has a particular risk for reverse causation-that is, individuals with lung disease or respiratory tract infections are more likely to remain indoors and consequently have lower vitamin $D$ synthesis. However, data from the 2 trials cited by Dr Hemilä, ${ }^{4,5}$ which did not find a reduction of respiratory tract infection with a vitamin D dose of $200 \mathrm{IU} / \mathrm{d}$ or lower, are notably limited by the low vitamin D dose contained in these supplements.

Conversely, a randomized controlled trial of bone loss in postmenopausal black women found that $7.7 \%$ of women randomized to vitamin D supplementation, 800 to $2000 \mathrm{IU} / d$, reported respiratory symptoms over the 3-year follow-up, compared with $25.0 \%$ in controls. ${ }^{6}$ Another substudy of a randomized controlled trial for fracture prevention found 
a non-statistically significant reduction in wintertime infection in participants randomized to vitamin D supplementation, $800 \mathrm{IU} / \mathrm{d}$ (adjusted odds ratio, 0.90; 95\% confidence interval, 0.76-1.07])..$^{7}$ Although promising, these studies were post hoc analyses of adverse events reported in skeletal health trials. Since serum 25-hydroxyvitamin D levels in the US population are relatively low, ${ }^{8}$ vitamin $D$ supplementation of at least 1000 to $2000 \mathrm{IU} / \mathrm{d}$ would be needed to raise levels above 30 to $40 \mathrm{ng} / \mathrm{mL}$ (to convert to nanomoles per liter, multiply by 2.496), which we believe is necessary for improved immune function and reduction of respiratory tract infection. A recently published pilot randomized controlled trial of 2000-IU/d vitamin D did not find a reduction of respiratory tract infections, but it was significantly limited by small sample size, delayed enrollment, incomplete recruitment, and high baseline serum 25-hydroxyvitamin D levels. ${ }^{9}$ We are planning larger randomized controlled trials in high-risk populations to understand whether the observed association between vitamin $D$ and respiratory tract infection is causal and reversible.

\section{Adit A. Ginde, MD, MPH \\ Jonathan M. Mansbach, MD \\ Carlos A. Camargo Jr, MD, DrPH}

Correspondence: Dr Camargo, Emergency Medicine Network, Massachusetts General Hospital, 326 Cambridge St, Ste 410, Boston, MA 02114 (ccamargo@partners .org).

1. Douglas RM, Hemilä H, Chalker EB, Treacy B. Vitamin C for preventing and treating the common cold. Cochrane Database Syst Rev. 2007;(3):CD000980.

2. Lawlor DA, Davey Smith G, Bruckdorfer KR, Kundu D, Ebrahim S. Those confounded vitamins: what can we learn from the differences between observational versus randomized trial evidence? Lancet. 2004:363(9422):1724-1727.

3. Ginde AA, Mansbach JM, Camargo CA Jr. Association between serum 25 hydroxyvitamin D level and upper respiratory tract infection in the Third $\mathrm{Na}$ tional Health and Nutrition Examination Survey. Arch Intern Med. 2009;169 (4):384-390

4. Liu BA, McGeer A, McArthur MA, et al. Effect of multivitamin and mineral supplementation on episodes of infection in nursing home residents: a randomized, placebo-controlled study [correction published in J Am Geriatr Soc. 2007;55(3):478]. J Am Geriatr Soc. 2007;55(1):35-42

5. Graat JM, Schouten EG, Kok FJ. Effects of daily vitamin E and multivitaminmineral supplementation on acute respiratory tract infections in elderly persons: a randomized controlled trial. JAMA. 2002;288(6):715-721.

6. Aloia JF, Li-Ng M. Re: epidemic influenza and vitamin D [letter]. Epidemiol Infect. 2007;135(7):1095-1098.

7. Avenell A, Cook JA, Maclennan GS, Macpherson GC. Vitamin D supplementation to prevent infections: a sub-study of a randomised placebo-controlled trial in older people (RECORD trial, ISRCTN 51647438). Age Ageing. 2007; 36(5):574-577.

8. Ginde AA, Liu MC, Camargo CA Jr. Demographic differences and trends of vitamin D insufficiency in the US population, 1988-2004. Arch Intern Med. 2009;169(6):626-632

9. Li-Ng M, Aloia JF, Pollack S, et al. A randomized controlled trial of vitamin D3 supplementation for the prevention of symptomatic upper respiratory tract infections [published online ahead of print March 19, 2009]. Epidemiol Infect. doi:10.1017/S0950268809002404.

\section{Fluconazole Use as an Important Risk Factor in the Emergence of Fluconazole- Resistant Candida glabrata Fungemia}

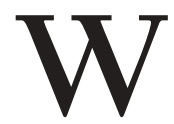

e read with interest the article by Lee et al, who conducted a case-case-control study to evaluate risk factors for fungemia caused by fluconazole-resistant Candida glabrata. The authors identified previous fluconazole use (and linezolid use) as a significant risk factor for fluconazole-resistant $C$ glabrata fungemia, whereas cefepime and metronidazole use were independent risk factors for fluconazolesusceptible $C$ glabrata fungemia.

Although with a smaller number of patients, a similar investigation with the same study design was previously carried out by our group. ${ }^{2}$ In accordance with Lee et al, ${ }^{1}$ our study had identified prior fluconazole exposure as the most important predisposing factor for subsequent $C$ glabrata fungemia due to a less-susceptible isolate. We found that diabetes mellitus and central venous catheters were other independent risk factors for fungemia caused by a fluconazole-less-susceptible isolate, whereas previous surgery and central venous catheterization were independent risk factors for fungemia due to a susceptible isolate; unfortunately, these variables do not appear to have been analyzed by Lee et al. ${ }^{1}$ With regard to the antibiotic use, we did not find a significant association between fluconazole-resistant $C$ glabrata fungemia and the use of any antibiotics; it was not possible to assess the impact of linezolid use because this drug was not largely used in Italy during the first years of our study. We found that cephalosporin (and glycopeptide or aminoglycoside) use was significantly associated with fluconazole-susceptible $C$ glabrata fungemia, but in univariate analysis only.

We agree with the authors' hypothesis that previous fluconazole use may promote de novo resistance through the CgPDR1-dependent ${ }^{3}$ upregulation of drug efflux pump-encoding genes CgCDR1 and CgCDR2 and also CgSNQ2. ${ }^{4}$ In fact, in our study, ${ }^{2}$ expression of these genes was upregulated in both the fluconazoleresistant and fluconazole-susceptible-dose-dependent isolates, but not in the fluconazole-susceptible isolates, making it likely that their reduced fluconazole susceptibility was acquired or induced following drug exposure. Our recent findings ${ }^{5}$ showed that CgPDR1 hyperactive alleles not only conferred a selective growth advantage compared with the wildtype allele in the murine model of systemic candidiasis in the absence of fluconazole, but also contributed to fluconazole treatment failure in the mouse model.

Finally, in our study, ${ }^{2}$ we observed that 6 of 7 patients receiving inadequate initial antifungal treatment died, and 5 of these patients were infected by a fluconazole-resistant isolate and empirically treated with fluconazole, supporting the conclusion that reduced susceptibility of $C$ glabrata to fluconazole contributes to a poor outcome after invasive infection, especially in conjunction with inadequate empirical antifungal treatment. When considering the larger population size studied by Lee et al, ${ }^{1}$ it would have been interesting if they had extended their analysis to this important issue.

\section{Mario Tumbarello, MD Brunella Posteraro, PhD Enrico M. Trecarichi, MD Maurizio Sanguinetti, MD}

Correspondence: Dr Posteraro, Istituto di Microbiologia, Università Cattolica del Sacro Cuore, Largo F. Vito 1,00168 Roma, Italy (bposteraro@rm.unicatt.it). 